\title{
Hybrid Predictive Control of Supermarket Refrigeration Systems: an Optimal Time Switching Strategy
}

\author{
D. Sarabia* C. de Prada* \\ * Dpt. Systems Engineering and Automatic Control, University of Valladolid \\ c/Real de Burgoss/n, 47011, Valladolid,Spain (dsarabia@autom.uva.es; prada@autom.uva.es)
}

\begin{abstract}
This paper presents a NMPC of a supermarket refrigeration system. This is a hybrid process involving switched nonlinear dynamics and discrete events, on/off manipulated variables (valves and compressors), continuous controlled variables (goods temperatures) and finally, several operation constraints. The hybrid controller is based on a parameterization of the on/off control signals in terms of time of occurrence of events instead of using directly binary values, in this way, we can re-formulate the optimization problem as a NLP problem. Results of the hybrid controller operation are given.
\end{abstract}

\section{INTRODUCTION}

A supermarket refrigeration system consists of a central compressor bank that maintains the required flow of refrigerant to the refrigerated display cases located in the sales area for self-service where goods are kept cooled. Each display case has an inlet valve for refrigerant that needs to be opened and closed such that the air temperature in the display case is kept within tight bounds to ensure a high quality of the goods.

A simplified layout of a system with two display cases is given in Fig. 1 while the cross section of a display case with its evaporator system is given in Fig. 2. The case study corresponds to an industrial system and was provided by Danfoss (DK) as a case study for the EU NoE HYCON. The compressor rack creates a low pressure in the suction manifold, which helps the evaporation of the refrigerant in the evaporators, with the corresponding transfer of heat from the display case wall to the refrigerant $Q_{e}$, which is the basic mechanism for cooling the air around the refrigerated goods. The compressed refrigerant is condensed and sent back to the evaporators, which are fed in parallel from the liquid manifold. The outlets of the evaporators lead to the suction manifold and back to the compressors thus closing the circuit.

The system is subjected to significant disturbances, like the strong transitions from day to night operation, that take place daily. The dynamic of the process is inherently continuous and, in principle, continuous control valves and variable speed compressors could be employed, nevertheless, instead, inlet on/off valve to every display case and start/stop compressors are utilized due to several practical reasons, which makes the control problem a hybrid one: continuous dynamics and controlled variables and on/off actuators.

These kind processes can be modelled in a set of inequalities, using for example the Mixed Logical Dynamical (MLD) framework (Bemporad and Morari, 1999). This leads, in the context of Model Predictive Control (MPC), to a mixed integer optimization problem that must be solved every sampling time, see, for instance (Larsen et al., 2005). This kind of formulation in the discrete time domain using lineal models allows for a systematic controller design, but in practice, due to the high dimensionality of the problem, except in a limited number of cases, it is not possible to solve the associated MILP or MIQP optimization problem on-line, even with multi-parametric methods. Additionally, linear models may not provide an adequate representation of the non-linear dynamics that takes place in refrigeration systems.

This paper, presents an alternative approach of the hybrid controller where on/off actions (valves and compressors) are formulated in terms of time instants of occurrence of events, instead of as binary values on each sampling time. The controller uses the framework of non-linear model predictive control, and uses a nonlinear continuous-time model instead to a discrete one, to compute the output predictions. The control actions are calculated by means of a dynamic optimization problem solved on-line every sample time. This approach has been tested by the authors in other applications and has the advantage that all decision variables are of real type, and its number is reduced significantly. Here, previous work (Sarabia et al., 2007) is extended to a more efficient control of the compressors, and using a more elaborated first principles model of the refrigeration system with improved computation capabilities.

A similar approach with two levels in the control architecture and NMPC framework is used in (Sonntag et al., 2007) but there discrete variables associated with changes of the state of the compressors are used solving a NLP problem for each of the combinations, and then choosing the best solution.

\section{TRADITIONAL CONTROL}

The traditional control of the supermarket refrigeration systems is decentralized. It is based on air temperature hysteresis control in each evaporator manipulating the on/off input valves, plus PI control of the suction pressure, with a 
quantifier on its output to determine the number of compressors in operation, see Fig. 1. This type of control, while cheap and reliable, give rise to a phenomenon of synchronization in the operation of the display cases, that increases the frequency of actuation of the compressors, limiting its life and creating picks of energy demand instead of a more evenly distributed one. This is the main problem to be avoided.

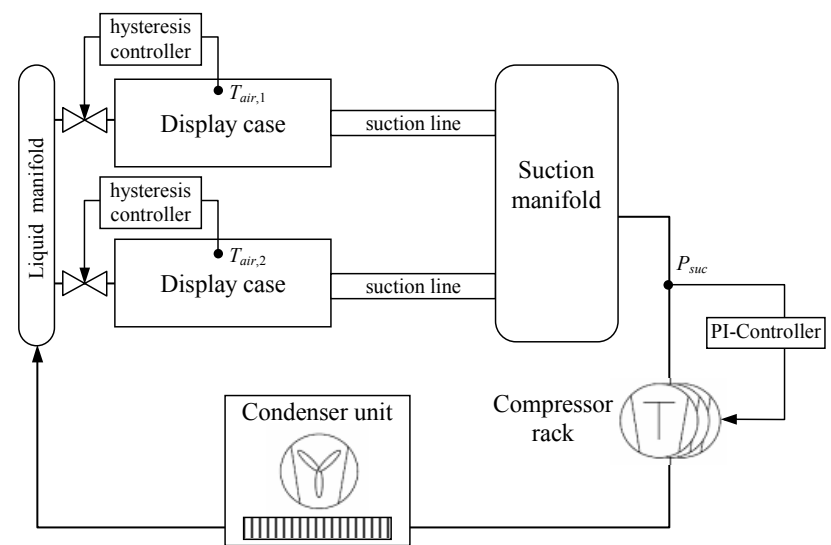

Fig. 1. A schematic of a supermarket refrigeration system.

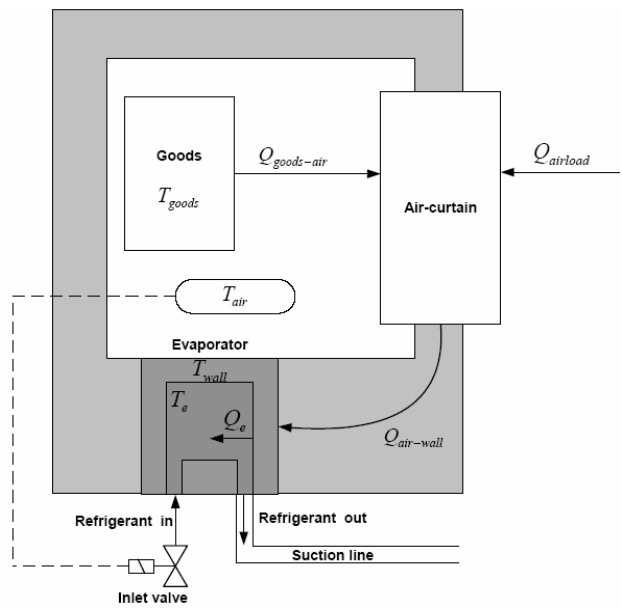

Fig. 2. Cross section of a refrigerated display case.

Results for the operation of a system with two display cases and two compressors, using this type of control, are given in Fig. 3, for a period covering day and night hours, where the synchronization of the oscillations in air temperatures can be seen clearly after an initial period were the oscillations are decoupled. The upper graph displays the time evolution of the air temperatures. Notice in the middle graph how the oscillation in the suction manifold pressure increases when the synchronization takes place. Then, in order to maintain the suction pressure, the controller has to change more frequently the number of compressors in operation as can be seen in the lower graph $(100 \%=2$ compressors operating, $50 \%$ only one and $0 \%$ none of them).

However, the hysteresis control has also positive side-effects. With this kind of control, the evaporator in the display cases is operated with a high degree of filling, so that, the temperature distribution across the display case will be homogenous, ensuring the all goods quality. This implies that in the off periods the frost will melt, so defrosting of the evaporators can be carried out less often. Normally, humid air will cause a frost built up on the coldest areas of the evaporator, thus deteriorating the heat transfer and blocking the air flow through the evaporator.
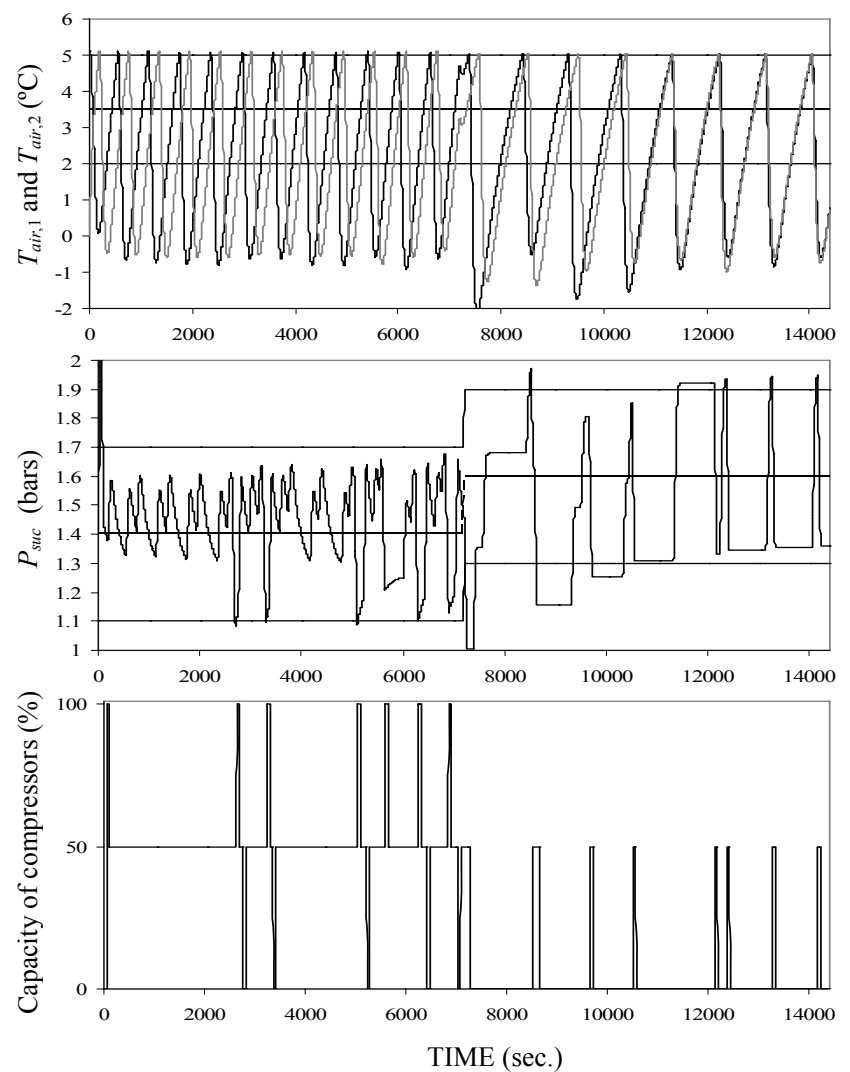

Fig. 3. Effects of synchronization on a) air temperatures $\left(T_{a i r, 1}\right.$ in black and $T_{a i r, 2}$ in grey) and b) on the suction pressure $\left(P_{s u c}\right)$ using traditional control. c) Discrete capacity of the compressors in percentage.

\section{SUPERMARKET MODEL}

The nonlinear model of the supermarket refrigeration system is composed of individual models for the display cases, the suction manifold, the compressor rack and the condensing unit (Larsen, 2007) and (Larsen et al., 2007). The refrigerant is led into the evaporator located at the bottom (see Fig. 2) of the display case number $i(i=1,2, \ldots, n d$ with $n d$ the total number of display cases), where the refrigerant evaporates while absorbing heat from the wall $Q_{e, i}$ and from the surrounding air circulating around the evaporator $Q_{\text {airload }}$. The resulting airflow creates an air-curtain at the front of the display case, that leads to a heat transfer $Q_{\text {goods-air, } i}$ from the goods to the air-curtain. Inside the display cases, a temperature sensor is located, which measures the air temperature $T_{a i r, i}$ close to the goods. This temperature is used in the control loop as an indirect measure of the goods' temperature $T_{\text {goods, } i}$ which is not measured directly and an on/off valve $u_{\text {disp, } i}$ is located at the refrigerant inlet of the evaporator. The dynamic in each display case is also described by the temperature of the evaporator wall $T_{\text {wall, } i}$ and the mass of liquefied refrigerant in the evaporator $M_{r e f, i}$. By setting up the energy balance for the goods, the air 
temperature and temperature of wall, the following three state equations can be derived,

$$
\begin{aligned}
& \frac{d T_{\text {goods }, i}}{d t}=-\frac{Q_{\text {goods-air }, i}}{M_{\text {goods }, i} C p_{\text {goods }, i}} \\
& \frac{d T_{\text {wall }, i}}{d t}=\frac{Q_{\text {air }- \text { wall }, i}-Q_{e, i}}{M_{\text {wall }} C p_{\text {wall }}} \\
& \frac{d T_{\text {air }, i}}{d t}=\frac{Q_{\text {goods-air }, i}+Q_{\text {airload }}-Q_{\text {air }- \text { wall }, i}}{M_{\text {air }} C p_{\text {air }}} \\
& Q_{\text {goods-air }, i}=U A_{\text {goods-air }}\left(T_{\text {goods }, i}-T_{\text {air }, i}\right) \\
& Q_{\text {air-wall }, i}=U A_{\text {air-wall }}\left(T_{\text {air }, i}-T_{\text {wall }, i}\right) \\
& Q_{e, i}=U A_{\text {wall-ref }, i}\left(M_{r e f, i}\right)\left(T_{\text {wall }, i}-T_{e, i}\right)
\end{aligned}
$$

where $U A$ is the heat transfer coefficient times surface with the subscript denoting the media between which the heat is transferred, $M$ denotes the mass, and $C p$ the heat capacity, where the subscript also denotes the media ("wall", "air" and refrigerant "ref"). $T_{e, i}$ is the evaporation temperature and are assumed equal for all evaporators. $Q_{e, i}$ is the cooling capacity, and, as indicated in (6), the heat transfer coefficient between the refrigerant and the evaporator wall $U A_{\text {wall-ref }, i}$ is a function of the mass $M_{\text {ref,i } i}$ of the liquefied refrigerant in the evaporator, and can be described by the following linear function:

$$
U A_{\text {wall-ref }, i}\left(M_{\text {ref }, i}\right)=U A_{\text {wall-refrig, } \max } M_{\text {ref }, i} / M_{\text {ref,max }}
$$

where $M_{\text {ref }, i}=M_{\text {ref, } \max }$ when the evaporator is completely filled with refrigerant. The accumulation of it in the evaporator follows the next switched nonlinear dynamic,

$$
\frac{d M_{r e f, i}}{d t}=\left\{\begin{array}{cl}
\left(M_{r e f, \max }-M_{r e f, i}\right) / \tau_{\text {fill }} & \text { if } u_{\text {disp }, i}=1 \\
-Q_{e, i} / \Delta h_{l g} & \text { if } u_{\text {disp }, i}=0
\end{array}\right.
$$

where $\tau_{\text {fill }}$ is a parameter describing the filling time of the evaporator when the valve is opened $u_{\text {disp }, i}=1$. However, when the valve is closed $u_{\text {disp }, i}=0$, the refrigerant is evaporated. If the evaporator is completely empty, then $M_{r e f, i}=0$ and $Q_{e, i}=$ 0 . The on/off manipulated variables are $u_{\text {disp }, i}(i=1,2, \ldots, n d)$ where $n d$ is the total number of display cases and $\Delta h_{l g}$ is the specific latent heat of the remaining liquefied refrigerant in the evaporator. When the refrigerant leaves the evaporator flows on to the suction manifold and the mass flow $f_{i}$ out of each evaporator can be computed as $f_{i}=Q_{e, i} / \Delta h_{l g}$.

Setting up the mass balance in the suction manifold, the corresponding state equation is obtained,

$$
\frac{d P_{s u c}}{d t}=\frac{f_{\text {in }-s u c}+f_{\text {ref }, \text { const }}-F_{\text {comp }} \rho_{\text {suc }}}{V_{\text {suc }} d \rho_{\text {suc }} / d P_{\text {suc }}}
$$

where $P_{s u c}$ is the suction pressure, $V_{s u c}$ and $\rho_{s u c}$ are the total volume of the suction manifold and the density of the refrigerant in it, $F_{\text {comp }}$ is the volume flow out of the suction manifold determined by the compressors, $f_{\text {in-suc }}=\sum_{i=1}^{n d} f_{i}$ is the total mass flow from the display cases to the suction manifold. $f_{\text {ref,const }}$ is a constant mass flow into the manifold originated from other un-modelled refrigerated entities, such as cold storage rooms.

In most refrigeration systems, the compressor capacity is discrete, so the compressors can be switched only either on or off. Let $n c$ denote the total number of compressors. The compressor bank can be modelled using a constant volumetric efficiency $\eta_{v o l}$ and the maximal displacement volume $V_{s l}$. Thus, the volume flow $F_{c o m p, i}$ out of the suction manifold created by the $i$ 'th compressor can be calculated as:

$F_{\text {comp }, i}=u_{c o m p, i} C_{c o m p, i} \frac{1}{100} \eta_{v o l} V_{s l} \quad i=1,2, \ldots \ldots, n c$

where $C_{\text {comp }, i}$ is the $i$ 'th compressor capacity $\left(\sum_{i=1}^{n c} C_{c o m p, i}=100\right)$, and $F_{c o m p}=\sum_{i=1}^{n c} F_{c o m p, i}$ is the total volume flow and, finally, $u_{\text {comp }, i}$ is the on/off manipulated variable associated with each compressor $i(i=1,2, \ldots, n c)$.

The model described before contains some non-linear refrigerant depended properties of suction pressure, for example, $T_{e}, \Delta h_{l g}, d \rho_{s u c} / d P_{s u c}$ and $\rho_{s u c}$, these properties are computed by using the freeware software package "RefEqns" (Skovrup) available for language $\mathrm{C}, \mathrm{C}++$, Matlab and others, incorporating it in our simulation of the process.

\section{CONTROL PROBLEM}

The control challenge of this supermarket refrigeration system is developing an efficient and optimal control strategy that avoids the synchronization effect on air temperatures (maintaining the others positive effects of hysteresis control) and optimizes the operation of the plant. Next, a brief summary of the control variables and the corresponding control objectives is presented.

The controlled variables are the suction pressure $P_{s u c}$ and the air temperature $T_{a i r, i}$ in each display case $(i=1, \ldots, n d)$. The sensors are indicated in Fig. 1.

The on/off manipulated variables are the start/stop action on the compressors $\left(u_{\text {comp }, i} \forall i=1, \ldots, n c\right)$ and the opening/closing of the inlet valves $\left(u_{\text {disp }, i} \forall i=1, \ldots, n d\right)$.

The measured disturbances are the heat load on the display cases $Q_{\text {airload }}$ and the additional flow of refrigerant into the suction manifold $m_{r e f, c o n s t}$. The mass of goods $M_{\text {goods,i }}$ in each display case is also assumed constant and known.

In principle, the only measured state is the temperature of the wall in each display case $T_{\text {wall, } i}$ while the others states are not measured. We have also assumed the goods temperature $T_{\text {goods }, i}$ and the mass of the refrigerant $M_{\text {ref }, i}$ as measured 
states, alternatively, they could be estimated. The air temperature, like in real process, is used to control indirectly the temperature of goods.

The overall control objectives are: (i) maintain the suction pressure below a maximum value $P_{s u c} \leq P_{\text {suc }}^{\text {ind } \max }$, (ii) maintain the goods temperatures in the display case within the specified temperature bounds. That is, the air temperatures have to be within the limits, $T_{a i r, i}^{\text {ind } \min } \leq T_{a i r, i} \leq T_{a i r, i}^{\text {ind } \max }$ and (iii) minimize the number of compressor switches. Reducing this number the lifetime of the compressors can be prolonged.

\section{HYBRID MODEL PREDICTIVE CONTROL}

A natural approach to many decision making problems is Model Predictive Control (MPC): An internal model of the process is used to predict its future behavior as a function of the present and future control actions, which are selected in order to minimize some performance index or cost function $J$. The optimal control signals corresponding to the present time are applied to the process and the whole procedure is repeated every sampling period. However, these concepts require certain adaptation in order to deal with hybrid systems. Next, a description of the components of the proposed Hybrid MPC is presented.

\subsection{Optimal time switching strategy}

The internal model used in the hybrid MPC is of continuous nature and is based on the first principles one presented in section 3. However, it is formulated in a different way. There, all manipulated variables were integers, corresponding to the open/close valve position in each display case $u_{d i s p, i}(t)$ and the start/stop of each compressor $u_{\text {comp }, i}(t)$ along the prediction horizon. Hence, the optimization of a certain cost function $J$ in terms on these variables implies solving a MINLP optimization problem every sampling time which is very costly to solve in real time. Nevertheless, we can avoid the use of these integer variables associated to the valve or compressor state in each sampling instant along the control horizon by means of a new parameterization converting the integer decision variables into real decision ones that correspond to the time instants $t_{i}$ when the binary actuator changes its state, as in Fig.4. This approach, besides reducing the number of manipulated variables by unlinking it of the number of sampling periods in the prediction horizon, allows using conventional non lineal optimization techniques (in terms only on real variables) instead of mixed-integer non lineal programming, decreasing the complexity of the optimization procedure and saving computation time.

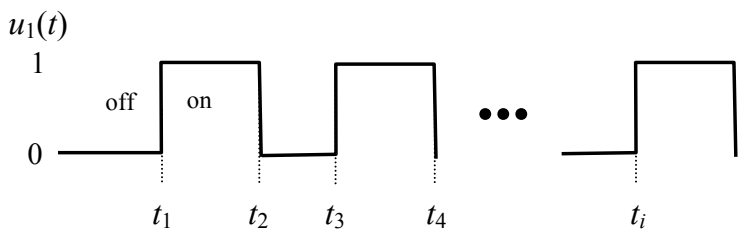

Fig. 4. Parameterization of integer decision variable $u_{1}(t)$.
In the following, and for simplicity, we will combine in a single manipulated variable $u_{i}$ the ones of the valves and compressors, with an index $i$ ranging from 1 to $n d$ and from $n d+1$ to $n d+n c$, where, the first $n d$ variables are the display case valves, named before $u_{d i s p, i}$, and the second $n c$ variables are associated with the compressors, that is $u_{c o m p, i}$.

Alternatively, we can define another parameterization using two new real variables $T_{o n, i}^{k}$ and $T^{k}{ }_{\text {off }, i}(k \in \mathbb{N})$ for each pulse of every integer manipulated variable $u_{i}(i=1, \ldots, n d+n c)$. denoting the time period for which this variable $u_{i}$ is one and when it is zero respectively for each pulse $k$, see Fig. 5 . The times $T_{o n, i}^{k}$ and $T_{o f f, i}^{k}$ are unknown variables that must be estimated along the control horizon.

\subsection{Predictions and Control Horizons}

The concept of control horizon $N_{u}$ in continuous MPC corresponds to the time interval where present and future control actions are computed. After $N_{u}$ sampling times, the controller maintains the last control signal computed at $N_{u}$ until the end of the prediction horizon $\mathrm{N}_{2}$ in order to compute the output predictions. In this hybrid environment, and referring to binary manipulated variables, it is more convenient to formulate the control horizon $N_{b, i}$ as the number of pulses of each control variable $u_{i}$ for which the controller compute the value of the decision variables $T^{k}{ }_{\text {on,i }}$ and $T_{\text {off }, i}^{k}$, (on and off periods of every pulse $k=1,2,3, \ldots$ ). From the pulse number $N_{b, i}$ until the end of the prediction horizon, the values of the decision variables $T_{o n, i}^{k}$ and $T_{\text {off }, i}^{k}$ will be taken equal to the ones of the pulse $N_{b, i}$, so, the schedule of $u_{i}$ will be forced to follow the pattern of the $N_{b, i}$ pulse, assuming that the system should reach a final stationary schedule.

This parameterization is based on the assumption that "the normal operation" of the manipulated variables toward the end of the prediction horizon, once the transient due to a disturbance or set point change has passed, will follow a periodical behavior along the time. Notice that a "stable" pattern of operation cannot be reach with a constant value of the actuators in this kind of processes, see for instance Fig 3.

Usually, the prediction horizon $N_{2}$ in MPC corresponds to the number of future sampling periods, of duration $T_{s}$, used to compute predictions with the internal model. In standard MPC, it is chosen longer than the process settling time. Notice that, as mentioned before, in our framework of on/off manipulated variables, the process will never settle in a stationary point, but will oscillate, eventually reaching a stable operational pattern. So, again, instead of specifying the interval where the predictions will be made, it is more convenient to talk in terms of $N_{p}$, number of pulses of the slower changing integer manipulated variable $u_{i}$, assumed to be required for obtaining a stable operational pattern. This means that, in order to compute a certain cost function $J$, the internal model will be integrated until the slowest train of pulses completes $N_{p}$ pulses. Notice that each integer manipulated variable can perform a different number of pulses in the same period of time $T_{p}$, see Fig. 5. 
Fig. 5 displays an example with two integer variables $u_{1}$ and $u_{2}$ and $N_{p}=4$ where the prediction horizon is the time needed by $u_{2}$ to implement four full pulses. We can also observe, that $u_{1}$ makes more pulses than $u_{2}$ (5 pulses). In this example, the variable $u_{1}$ has $N_{b, 1}=1$, so, only the times $T_{\text {on, } 1}^{1}$ and $T_{\text {off, }, 1}^{1}$, that correspond to the first pulse will be computed and the same pattern will be applied in the following ones, that is to say, $T_{o n, 1}^{k}=T_{o n, 1}^{1}$ and $T_{o f f, 1}^{k}=T_{o f f, 1}^{1} \forall k>N_{b, 1}=1$. Bellow, $u_{2}$ has been assigned $N_{b, 2}=2$, so, the controller computes $T_{\text {on, } 2}^{1}, T^{1}{ }_{\text {off }, 2}$, $T_{o n, 2}^{2}$ and $T_{\text {off }, 2}^{2}$ and from the second pulse on, it follows the pattern defined by the last $T_{\text {on, } 2}^{2}$ and $T_{\text {off, } 2}^{2}$ computed times, so $T_{o n, 2}^{k}=T_{o n, 2}^{2}$ and $T_{o f f, 2}^{k}=T_{o f f, 2}^{2} \forall k>N_{b, 2}=2$.

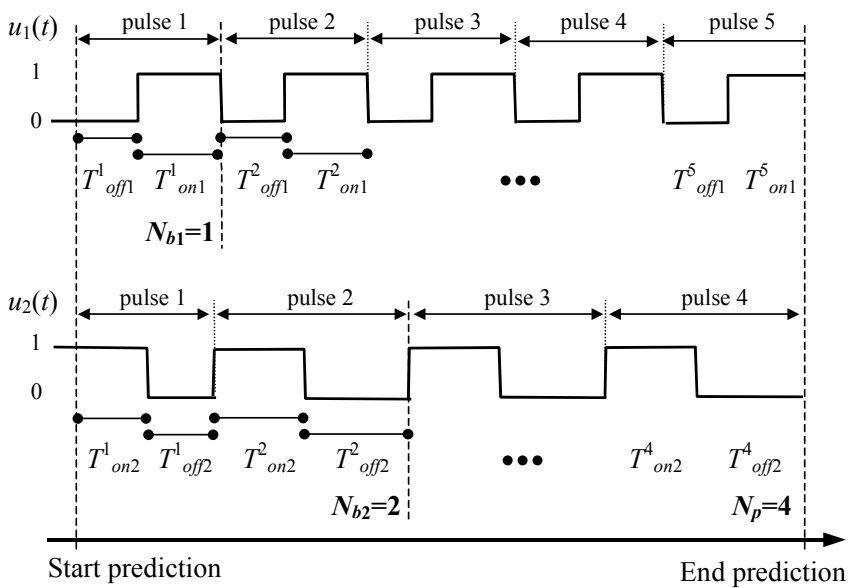

Fig. 5. Prediction $\left(N_{p}\right)$ and control horizons $\left(N_{b 1}, N_{b 2}\right)$ for integer variables $u_{1}$ and $u_{2}$ in hybrid MPC.

\subsection{The cost function}

The performance index $J$ to be minimized is the function:

$$
\begin{aligned}
J & =\int_{0}^{T_{p}} \alpha_{1}\left(S^{\max }\left(P_{\text {suc }}-P_{\text {suc }}^{\max }\right)^{2}+S^{\min }\left(P_{\text {suc }}-P_{\text {suc }}^{\min }\right)^{2}\right) d t \\
& +\sum_{i=1}^{n d} \alpha_{1+i} \sum_{\min \& \max }\left(\left(\hat{T}_{\text {air }, i}^{\max }-T_{\text {air }, i}^{r e f \max }\right)^{2}+\left(\hat{T}_{\text {air }, i}^{\min }-T_{\text {air }, i}^{\text {ref } \min }\right)^{2}\right) \\
& +\sum_{i=1}^{n c} \alpha_{1+n d+i} \sum_{k=1}^{N_{b, i}}\left(T_{\text {on }, 1+n d+i}^{k}\right)
\end{aligned}
$$

Where the first term in (11) is the brakets function penalizing the evolution of the suction pressure out of its range along the prediction, that is, $S^{\max }=1$ if $P_{s u c} \geq P_{\text {suc }}^{\max }$ or $S^{\max }=0$ otherwise, and $S^{\mathrm{min}}=1$ if $P_{\text {suc }} \leq P_{\text {suc }}^{\min }$ or $S^{\mathrm{min}}=0$ otherwise. If $P_{\text {suc }}^{\max } \leq P_{\text {suc }}^{\text {ind } \max }$ the first control objective in section 4 is fulfilled.

The minimization of the second term in (11) is a non conventional one that tries to obtain a stable periodical pattern of the air temperatures in the display cases: $\hat{T}_{a i r, i}^{\max }$ and $\hat{T}_{\text {air }, i}^{\min }$ are the maximum and minimum values reached by $T_{\text {air }, i}$ along the prediction, and they are calculated during each prediction. The idea is to obtain waves with a constant amplitude fixed by the maximum and minimum values allowed $T_{a i r, i}^{r e f \max }$ and $T_{a i r, i}^{r e f \min }$ (desired amplitude $=T_{a i r, i}^{r e f \max }$ $\left.T_{a i r, i}^{r e f \min }\right)$. Fig. 6 a) shows how to evaluate this term, assuming than the "natural" behavior of these variables is a periodical oscillation. Fig. 6 b) displays the differences between this kind of penalization and the typical one, in terms on the error between the controlled variable and its reference. Notice that, the values $T_{a i r, i}^{r e f \max }$ and $T_{a i r, i}^{\text {ref } \min }$ are the equivalent form in the periodical environment to the set-points in a classical continuous process. These variables are different than $T_{\text {air }, i}^{\text {ind } \max }$ and $T_{\text {air }, i}^{\text {ind } \min }$ described before in the section 4. This term also allow to increase the opening time of the valves, ensuring that the evaporators are filled with refrigerant to a high degree. Taking into account that $T_{\text {air }, i}^{\text {ref } \max } \leq T_{\text {air }, i}^{\text {ind } \max }$ and $T_{\text {air, }, i}^{r e f, \min } \geq T_{\text {air }, i}^{\text {ind } \min }$, if these "set-points" are followed, then the air temperatures are always within the extended range $\left[T_{\text {air }, i}^{\text {ind } \max }, T_{\text {air }, i}^{\text {ind } \min }\right]$ which is the second control objective in section 4 .

The third term in cost function $J$ tries to minimize the number of switches of each compressor along the prediction, by means on minimizing the all times in which compressors are on $\left(T_{o n, 1+n d+i}^{k}\right.$ with $\left.i=1, \ldots, \underline{n c}\right)$ corresponding with the third control objective in section 4. Finally, in (11) $\alpha_{i}$ $(i=1, \ldots, 1+n d+n c)$ are appropriate weight factors and $T_{p}$ is the total time of the prediction (equivalent to $N_{p}$ pulses).
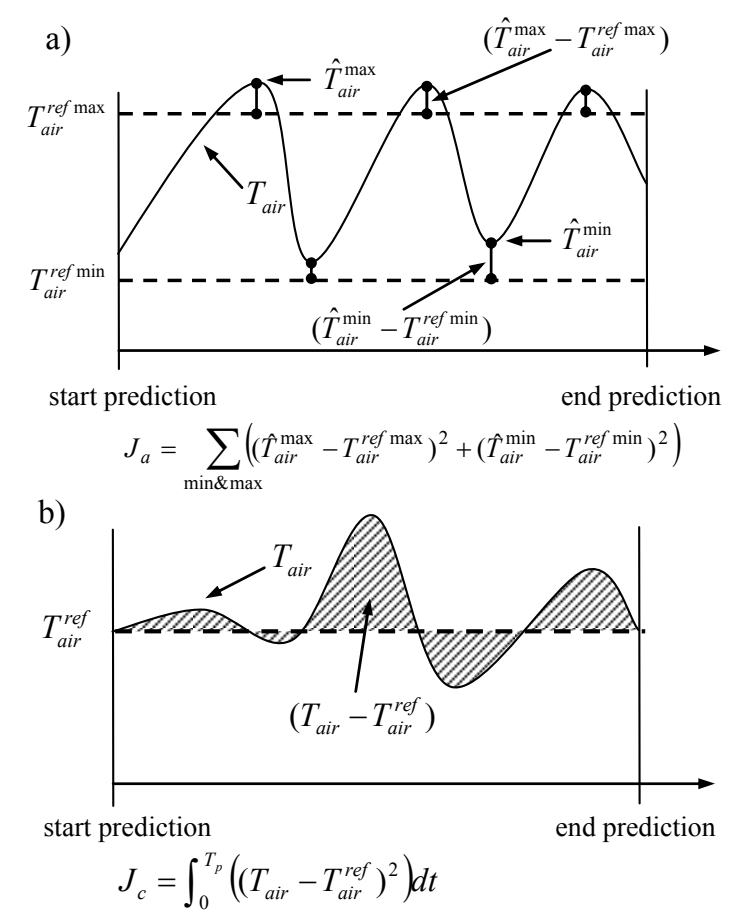

Fig. 6. a) Cost function associated with air temperatures when these variables have a periodical behavior. b) Classical cost function. 


\subsection{Architecture of the Hybrid MPC Controller}

The aim of Hybrid MPC is to minimize (11) under the dynamics (1)-(10) with relation to the decision variables $T^{k}{ }_{o n, i}$ and $T_{o f f, i}^{k}\left(k=1, \ldots, N_{b, i}\right.$ and $\left.i=1, . ., n d+n c\right)$ and satisfying static linear constraints on the decision variables:

$$
\begin{aligned}
& \left.\begin{array}{c}
T_{o n, i}^{\min } \leq T_{o n, i}^{k} \leq T_{o n, i}^{\max } \\
T_{o f f, i}^{\min } \leq T_{o f f, i}^{k} \leq T_{o f f, i}^{\max }
\end{array}\right\} \forall k=1, \ldots, N_{b, i} \forall i=1, \ldots, n d \\
& \left.\begin{array}{c}
0 \leq T_{o n, i}^{k} \leq T_{o n, i}^{\max } \\
0 \leq T_{o f f, i}^{k} \leq T_{o f f, i}^{\max }
\end{array}\right\} \forall k=1, \ldots, N_{b, i} \forall i=n d, \ldots, n c \\
& \left.\begin{array}{l}
T^{\min } \leq T_{o n, i}^{k}+T_{o f f, i}^{k} \leq T^{\max }
\end{array}\right\} \forall k=2, \ldots, N_{b, i} \forall i=n d, \ldots, n c
\end{aligned}
$$

As we can see in (12), the minimum value of the times associated to the compressors $\left(T_{\text {on, } i}^{k}\right.$ and $T_{\text {off } i}^{k}$ with $\left.i=n d, . ., n c\right)$ is set to zero. In this way, we allow the compressors not changing its state, that is, along a certain pulse $k$, one compressor can be always on or off, or on and off for a fraction of it. Notice that, at this respect, evaporator valves and compressors behave differently. Imposing pulses with all times different from zero is not necessarily the pattern that corresponds to the best compressor behaviour, creating solutions more "active" than necessary. As we will see in the next section, the optimal solution sometimes involves a periodical behaviour of the compressors and sometimes not. The last constraint in (12) guaranties that always exists a certain future pulse $k$ for each compressor.

The resulting optimization problem involves $\sum_{i=1}^{n d+n c} 2 \times N_{b, i}$ decision variables and is solved periodically, with a given sampling period using an SQP algorithm (implemented in a commercial library NAG for C) using a schematic like the one of Fig. 7, that corresponds to a sequential or control parameterization method (Sargent and Sullivan, 1977) where the cost function $J$ is computed by integration of the dynamical internal model. The simulation package integrates the internal model equations along the prediction horizon $N_{p}$ taking as initial conditions the current process state and evaluating the formulated cost function $J$ at the end of the integration. The internal model was simulated using the EcosimPro simulation language (EA, 1999) which allows combining DAE equations with events, while performing a correct integration in spite of the model discontinuities. It belongs to the category of the so called "modeling languages" that are object oriented and allows an easy re-use of the components. It generates automatically $\mathrm{C}++$ code corresponding to the simulation. This code of the internal model is embedded in the controller, which has been programmed in $\mathrm{C}++$.

Notice that the discontinuities in the model take place only at times when a manipulated variable changes its state, so that the computation of the gradients performed by SQP for each particular decision variable is made in such a way that the number or order of the discontinuities do not change along the prediction horizon, allowing a safe estimation of them. (Galan et al., 1999).
From a technical point of view, it is necessary to add a converter between the controller and the process. Hybrid MPC calculates the exact time instants $T_{\text {on, } i}^{k}$ and $T_{\text {off }, i}^{k}$ of changes for each on/off manipulated variable, and the converter transform these times in a discrete manipulated signal $u_{i}$, when the events will occur, see Fig. 7. Notice that this feature, along with the continuous nature of the internal model, allows decoupling the application of the on/off signals from the sampling time.

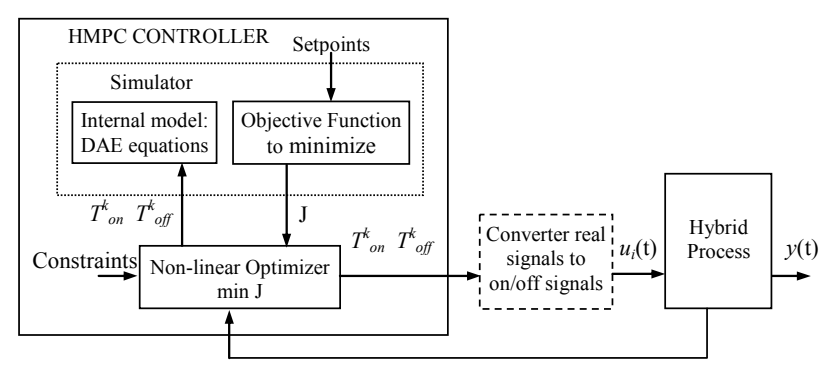

Fig. 7. Non-linear MPC controller implementation.

\section{SIMULATIONS AND CONTROL RESULTS}

The process model described in section 3 has been simulated and tested using the EcosimPro environment. To illustrate the behavior and performance of the hybrid MPC, we have made an experiment of 4 hours (14400 sec.) corresponding with a day/night operation ( 2 hours during the day and 2 hours during the night) of a supermarket refrigeration system. The refrigeration system has two compressor with the same capacity $C_{\text {comp }, 1}=C_{\text {comp }, 2}=50 \%$ and two equally sized display cases. In supermarkets and during the nights, the display cases are covered by "night-covers", hence effectively decreasing the load around $40 \%\left(Q_{\text {airload }}=3000 \mathrm{~J} / \mathrm{sec}\right.$ to 1800 $\mathrm{J} / \mathrm{sec}$ ) and reducing the constant mass flow into the suction manifold $\left(f_{\text {ref,const }}=0.2\right.$ to $\left.0.0 \mathrm{~kg} / \mathrm{sec}\right)$, being these known changes. As a result, the maximal suction pressure is allowed to increase $\left(P_{\text {suc }}^{\text {ind } \max }=1.7\right.$ bar to 1.9 bar). The upper and lower bounds for the air temperatures in all display cases remain unchanged between day and night, being set at $T_{\text {air }}^{\text {ind } \max }=5^{\circ} \mathrm{C}$ and $T_{\text {air }}^{\text {ind } \min }=2^{\circ} \mathrm{C}$. The initial values of the states (including the simulation with traditional control, Fig. 3) are $T_{\text {wall, }, 1}=$ $T_{\text {wall }, 1}=0.0{ }^{\circ} \mathrm{C}, T_{\text {air }, 1}=5.1{ }^{\circ} \mathrm{C}, T_{\text {air }, 2}=0.0^{\circ} \mathrm{C}, T_{\text {goods }, 1}=T_{\text {goods }, 2}=$ $4.0^{\circ} \mathrm{C}, M_{r e f, 1}=0.0 \mathrm{~kg}, M_{\text {ref }, 2}=1.0 \mathrm{~kg}$ and $P_{\text {suc }}=1.4$ bars.

Regarding the hybrid MPC controller, the sampling time was set at $60 \mathrm{sec}$., the prediction horizon was fixed in $N_{p}=3$, which corresponds to predictions of around 25 minutes (1500 sec.), and the control horizons for every manipulated integer variable were selected as $N_{b, 1}=N_{b, 2}=N_{b, 3}=N_{b, 4}=2$. So the optimization problem had 16 decision variables. Finally, the rest of parameters in cost function (12) are $P_{\text {suc }}^{\max }=1.6$ bars, and $P_{s u c}^{\min }=1.2$ bars during the day, changing these values during the night to 1.8 bars and 1.4 bars respectively. For both air temperatures $T_{\text {air }}^{\text {ref } \max }=4.8^{\circ} \mathrm{C}$ and $T_{\text {air }}^{r e f \min }=2.2^{\circ} \mathrm{C}$, with weights $\alpha_{1}=1.1$, and $\alpha_{2}=\alpha_{3}=\alpha_{4}=\alpha_{5}=1$. 
Fig. 8 and Fig. 9 show the evolution of the main variables The left part corresponds to day operation and the right hand side to night operation. Dotted lines in Fig. 7 show the upper and lower constraints on controlled variables defined in section 4. Notice that, in spite of the disturbance introduced by night conditions, the controller presents important improvements respect to the traditional control (Fig. 3): there is no synchronization on the air temperatures, the range of oscillation of the air temperatures and of the suction pressure have been decreased substantially and there is a reduction in the number of switches of the compressors, being only necessary use one compressor during all simulation, see lower graph of Fig. 9, where the capacity of the compressors

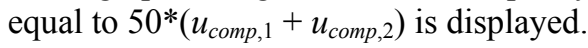

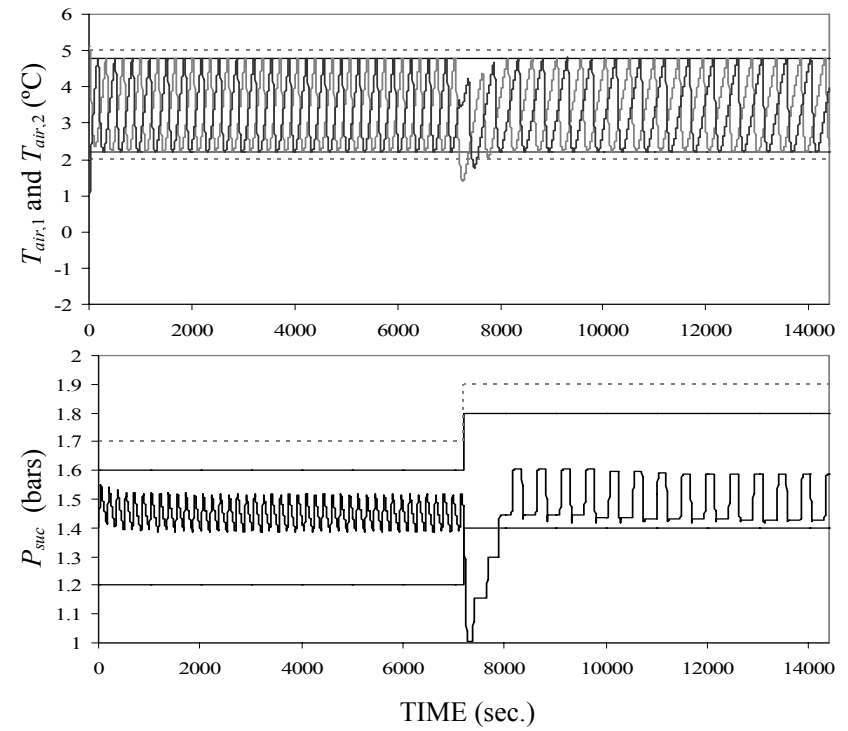

Fig. 8. Controlled variables: Air temperatures $\left(T_{a i r, 1}\right.$ in black and $T_{a i r, 2}$ in grey) and suction pressure $\left(P_{s u c}\right)$.
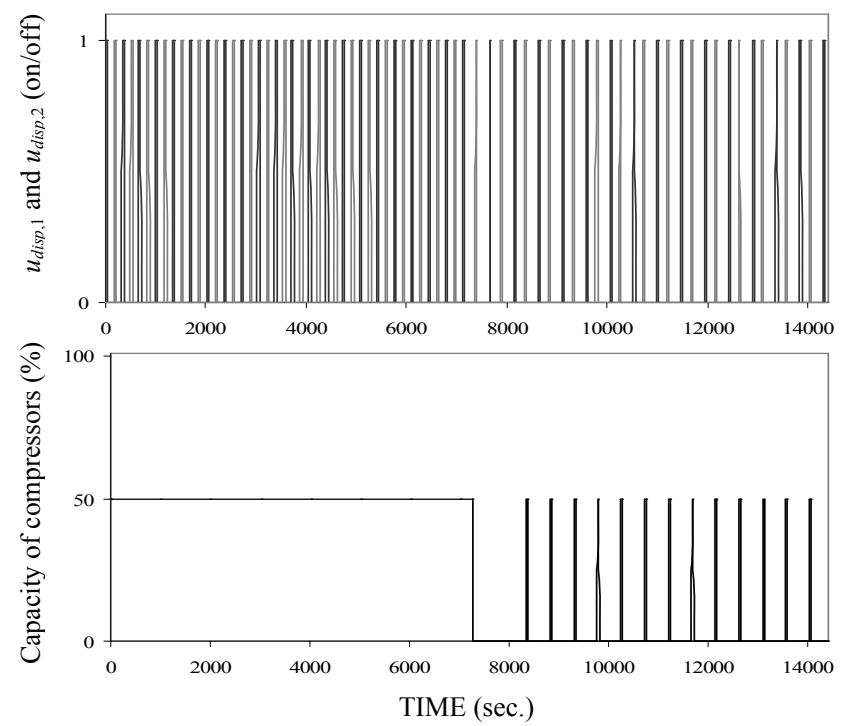

Fig. 9. Manipulated variables. The valves $\left(u_{\text {disp }, 1}\right.$ in black and $u_{\text {disp }, 2}$ in grey) and the capacity of the compressors.

The control approach followed in this paper (NMPC with continuous first principles internal model, parameterization of integer manipulated variables and forcing the integer variables to follow a periodical pattern at the end of the control horizon) presents good results on a realistic simulation of a supermarket refrigeration systems. In this approach, adding more devices (display cases or compressors) implies an increase on the decision variables but not in an exponential form (like in MPC with MLD models and integer decision variables, see Larsen et al., 2005), making possible the implementation of this controller in larger refrigeration systems. The operation in continuous time allows decoupling the formulation of the problem from the choice of the sampling period. This feature besides a two level architecture for the implementation of the optimal decisions of the hybrid controller, permits to use larger sampling times, being the control signals always applied in the corresponding exact instant calculated by the hybrid controller.

\section{ACKNOLEDGEMENT}

This work was supported in part by the Spanish Ministry of Education and Science (former MYCT) through project DPI2006-13593, as well as the European Commission through FP6 NoE HYCON, Contract no.: 511368.

\section{REFERENCES}

Bemporad A. and M. Morari (1999). Control of systems integrating logic, dynamic, and constraints. Automatica, 35, 407-427.

Galán, S., F. William and P.I. Barton (1999), Parametric sensitivy functions for hybrid discret/continuous systems. Applied Numerical Mathematics, 31(1), 17-47.

EA Int. (1999). EcosimPro User Manual. Available: http://www.ecosimpro.com.

Larsen, L.F.S., T. Geyer and M. Morari (2005). Hybrid MPC in supermarket refrigeration systems. In 16th IFAC World Congress, Prague, Czech Republic.

Larsen, L.F.S. (2007). Model Based Control of Refrigeration Systems. PhD thesis, Department of Control Engineering, Aalborg University, Aalborg, Denmark.

Larsen L.F.S., R. Izadi-Zamanabadi and R. Wisniewski (2007). Supermarket Refrigeration System-Benchmark for Hybrid System Control. Internal report; Available: http://www.control.aau.dk/hybrid/index.php?id=272.

Sarabia D., F. Capraro, L. F. S. Larsen and C. de Prada, (2007). Hybrid Control of a Supermarket Refrigeration Systems. American Control Conference, ACC07, New York, USA.

Sargent, R.W.H. and G.R Sullivan (1977). The development of an efficient optimal control package. In Proc. 8th IFIP Conf. Optim. Tech., Pt.2.

Skovrup, M.J. Thermodynamic and Thermophysical Properties of Refrigerants. Software package in Borland Delphi, Version 3.00.

Sonntag C., A. Devanathan, S. Engell and O. Stursberg (2007). Hybrid Nonlinear Model-Predictive Control of a Supermarket Refrigeration System. IEEE MultiConference on Systems and Control (MSC), Singapore. 